\title{
Generic Techniques to Reduce SVC Enhancement Layer Encoding Complexity
}

\author{
Sebastiaan Van Leuven, Student Member, IEEE, Jan De Cock, Member, IEEE, Rosario Garrido-Cantos, \\ Student Member, IEEE, José Luis Martínez, Member, IEEE, and Rik Van de Walle, Member, IEEE
}

\begin{abstract}
Scalable video coding is an important mechanism to provide several types of end-user devices with different versions of the same encoded bitstream. However, scalable video encoding remains a computationally expensive operation. To decrease the complexity we propose generic techniques. These techniques are generic in a sense that they can be combined with existing fast mode decision methods and optimizations. We show that extending such an existing fast mode decision technique yields an average complexity reduction of $87.27 \%$, while only an additional $0.74 \%$ of bit rate increase and a decrease of $0.11 \mathrm{~dB}$ in PSNR is required, compared to the original fast mode decision method ${ }^{l}$.
\end{abstract}

Index Terms - Fast mode decision, Scalable Video Coding, Spatial enhancement layers, Low complexity encoding.

\section{INTRODUCTION}

In this fast evolving world, scalability is more than ever key to efficiently cope with this changing environment. For example users want to be able to watch television on an HDTV, mobile phone, computer with high bandwidth Internet connection or on a notebook with a low bandwidth wireless connection. Instead of delivering all these streams simultaneously in simulcast, scalability could reduce bandwidth and thus operational cost. Scalable Video Coding (SVC) exploits the redundant information between these streams.

Applications for SVC, an extension of H.264/AVC [1], have not yet met the market. One of the reasons is the significant increase of the encoding complexity over singlelayer H.264/AVC video, due to the layered nature of SVC. SVC allows for three types of scalability, i.e. quality, temporal and spatial [2]. Using quality scalability, additional quality information is transmitted to the user, while temporal

\footnotetext{
${ }^{1}$ The research activities that have been described in this paper were funded by Ghent University, the Interdisciplinary Institute for Broadband Technology (IBBT), Ph.D. and post-doctoral fellow grants of the Agency for Innovation by Science and Technology (IWT), the Fund for Scientific Research-Flanders (FWO-Flanders), and the European Union.

Sebastiaan Van Leuven, Jan De Cock, and Rik Van de Walle are with the Multimedia Lab research group, Department of Electronics and Information Systems (ELIS), Ghent University-IBBT, B-9050 Ledeberg-Ghent, Belgium. (e-mail:\{ sebastiaan.vanleuven; jan.decock; rik.vandewalle\}@ugent.be)

Rosario Garrido-Cantos is with the Albacete Research Institute of Informatics, University of Castilla-La Mancha, Albacete, Spain. (e-mail: charo@dsi.uclm.es)

José Luis Martínez is with the Architecture and Technology of Computing Systems Group, Complutense University, Ciudad Universitaria s/n, 28040 Madrid, Spain. (e-mail: joseluis.martinez@fdi.ucm.es)
}

scalability allows adapting the frame rate. Both techniques slightly increase complexity, in contrast to spatial scalability.

Spatial scalability allows different resolutions to be encoded in a single bitstream. In order not to end up with a simulcast scenario, where all streams are encoded independently, interlayer prediction (ILP) can be applied [3]. Using ILP, the lowest resolution (base layer) can be used as a predictor for higher resolutions (enhancement layers). Hence, the mode decision (including motion estimation) has to be performed twice for the enhancement layer, once using regular techniques (as in H.264/AVC) and once with the base layer as a predictor. Therefore, spatial scalability comes with a high complexity.

To reduce the encoding complexity of the enhancement layer, fast mode decision models have been proposed. Most of these models are based on limiting the evaluations of macroblock partition size, or inter-layer residual prediction. While many relevant methods are listed here, many more techniques have been proposed. The following overview of related work highlights the best performing and most important methods from the viewpoint of this paper.

A fast mode decision method exploiting neighboring macroblock statistics is proposed in [4]. This method reports a $44.81 \%$ time saving. A selective inter-layer residual prediction method reduces complexity with $40 \%$ [5]. This is achieved by evaluating all modes without inter-layer residual prediction and re-evaluating the rate-distortion (RD) optimal mode with inter-layer residual prediction. Finally, the RD optimal prediction is applied for the mode. Because for this technique all modes have to be evaluated, it could be used for improving existing fast mode decision models, as will be shown in Section III-C.

The previously mentioned methods do not exploit encoded base layer information, such as macroblock types, for the spatial enhancement layer mode decision. Doing so, lower complexities are possible as shown in [6], which uses a classification mechanism for the most probable modes, based on base layer information. This results in a complexity reduction of $65 \%$, with a reported bit rate increase of $0.17 \%$. Macroblock modes can be prioritized based on the base layer macroblock type, as is suggested by [7]. Based on the state (i.e., all-zero block) of the current macroblock and neighboring macroblocks, an early termination strategy is applied to the prioritized list. The reported small complexity reduction of $20.23 \%$ for CGS and $27.47 \%$ for dyadic spatial scalability makes this technique less suited as a stand-alone technique. However, in combination with other techniques this 
could yield a lower complexity. Another prioritizing scheme [8] alters the mode decision, based on the base and enhancement layer neighboring macroblocks, yielding a $30 \%$ time saving.

Li's model [9] limits the enhancement layer mode decision based on co-located base layer modes. An off-line analysis of encoded video streams determines which modes are not likely to be selected. This model shows significant time savings of $60 \%$ on average, with small bit rate and PSNR changes.

In a profound analysis [10], the authors identified the importance of both the quantization and base layer macroblock mode for the enhancement layer mode decision process. Based on these observations, the authors have proposed a method which also takes the quantization of both layers into account [11], achieving a complexity reduction of $75 \%$, while maintaining a high RD performance. Modes with a high probability for being selected in the enhancement layer are always evaluated. MODE_ $8 \mathrm{x} 8$ is optimized by reducing the sub-mode calculations. Furthermore, the concept of orthogonal macroblock modes is introduced. These are modes for which the direction of their partitioning is orthogonal (e.g.: MODE_8x16 vs. MODE_16x8). Doing so, this method accomplishes a complexity reduction of $75 \%$, while maintaining a high RD performance.

This analysis is used to derive the proposed generic techniques, which are applicable for other fast mode decision models. Some of these ideas have been used in [11]. However, general sub-mode decision optimizations will significantly improve any existing fast mode decision models.

In the next section, the proposed generic techniques are introduced, based on the analysis. In Section III, the results are given for applying the proposed techniques as standalone techniques and for the combination of Li's model with the proposed technique. Additionally, selective inter-layer residual prediction is applied to identify the universality of the generic techniques. Future work is identified in Section IV. Finally, Section V presents the conclusions.

\section{Proposed Generic Techniques}

The proposed generic techniques can be mutually combined and used in combination with most fast mode decision models, as long as the applicable (sub-)process is not already altered. For example, when a mode decision process alters the submode decision of MODE_8x8, our proposed sub8x8 optimization cannot be applied.

These techniques are derived from an analysis, based on 10 sequences from which 5 sequences (Bus, Football, Foreman, Mobile, and Stefan) have been encoded with a QCIF base layer resolution and a CIF enhancement layer resolution (noted as QCIF/CIF), and 5 sequences (City, Crew, Cyclists, Night, and Optis) have been encoded with a CIF resolution for the base layer, and a $4 \mathrm{CIF}$ resolution for the enhancement layer (noted as CIF/4CIF). Both resolutions obtain the same results, which indicate that the probabilities for enhancement layer modes are independent of the resolution. A range of quantization parameters $(Q P)$ for base layer $\left(Q P_{B L}\right)$ and

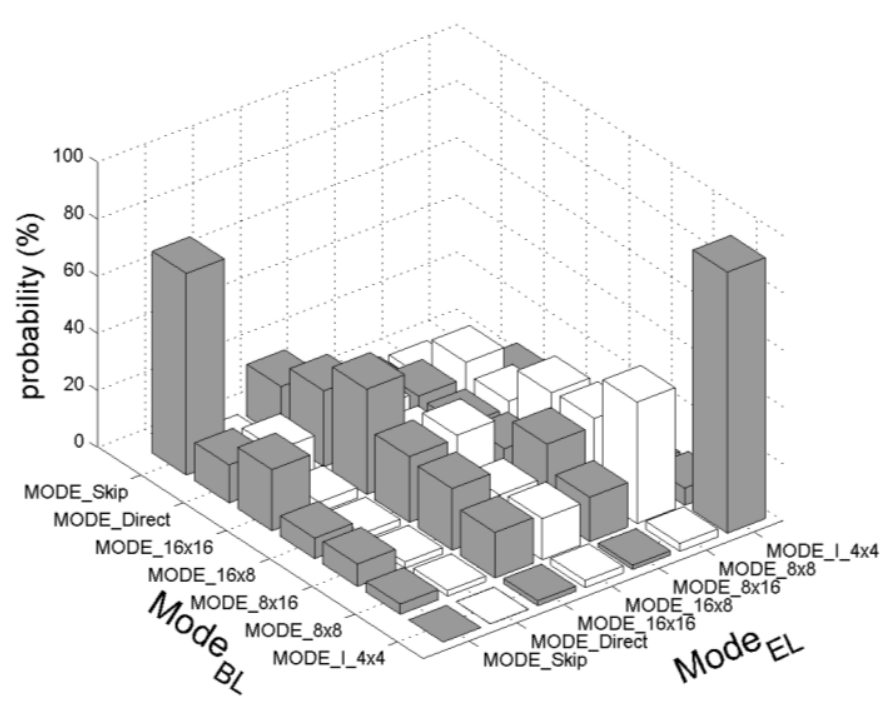

Fig. 1 Average conditional probabilities for enhancement layer modes $\left(\right.$ Mode $\left._{\mathrm{EL}}\right)$ based on the base layer mode (Mode $\left.{ }_{\mathrm{BL}}\right)$.

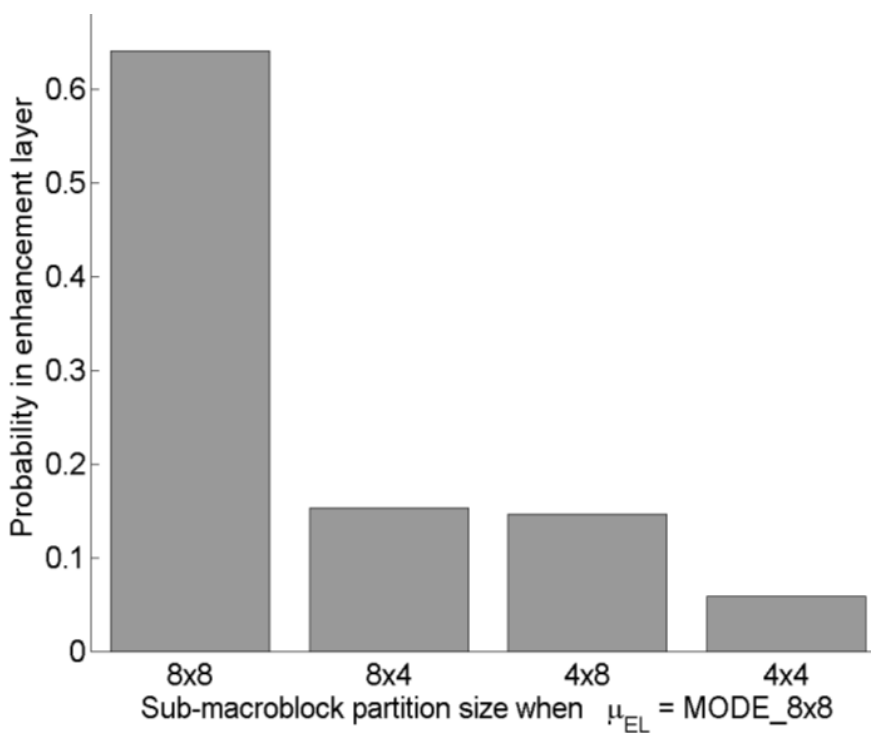

Fig. 2 Distribution of sub-macroblock partition sizes for MODE_8x8 in spatial enhancement layers.

enhancement layer $\left(Q P_{E L}\right)$ is applied: $Q P_{B L}, Q P_{B L} \in$ $\{12,15 \ldots 51\}$. For each combination of $\left(Q P_{B L}, Q P_{E L}\right), 64$ frames have been encoded using the Joint Scalable Video Model (JSVM) [12]. From the resulting encoded streams, the conditional probability that an enhancement layer macroblock type $\left(\mu_{\mathrm{EL}}\right)$ is selected, given the co-located base layer macroblock type $\left(\mu_{\mathrm{BL}}\right)$ is derived, which is expressed as $\mathrm{P}\left(\mu_{\mathrm{EL}} \mid \mu_{\mathrm{BL}}\right)$. Observations of the conditional probability lead to the following proposed generic techniques, which will be referred to as 1,2 , and 3 , respectively.

\section{1) Disallow orthogonal macroblock modes}

Fig. 1 shows for modes MODE_8x16 and MODE_16x8 that these have a low probability in the enhancement layer when the orthogonal mode has been selected for the co-located macroblock in the base layer. Therefore, the orthogonal mode of the base layer should not be evaluated during the enhancement layer mode decision process. 
TABLE I

AVERAGE COMPLEXITY OF SUB8X8-PARTITION SIZES

RELATIVE TO THE TOTAL MODE_8X8 COMPLEXITY

\begin{tabular}{|c|c|}
\hline Sub-macroblock partition size & Relative complexity (\%) \\
\hline Direct_8x8 8.05 \\
\hline $8 \times 8$ & 20.02 \\
\hline $8 \times 4$ & 22.66 \\
\hline $4 \times 8$ & 25.92 \\
\hline $4 \times 4$ & 31.35 \\
\hline
\end{tabular}

This observation makes sense since the visual content in both layers is highly correlated. Due to the higher resolution of the enhancement layer, the encoder will either select a less partitioned macroblock mode or the same macroblock partitioning direction.

2) Only evaluate sub8x8 blocks if present in base layer

As can be seen from Fig. 2, less than $40 \%$ of all MODE_8x8 macroblocks have a sub8x8-partition size (i.e., $8 \times 4,4 \times 8,4 \times 4)$. Meanwhile, almost $80 \%$ of the complexity of MODE $8 \times 8$ is required for those sub- $8 \times 8$ partitions, as shown in TABLE I. Therefore, sub8x 8 evaluation is limited to regions where they have been selected in the base layer. When a less partitioned macroblock mode is selected for the base layer, this indicates that less details are required to be encoded in the content. Since upscaling mostly preserves this property, it is unlikely that a finer partitioned macroblock mode will be selected in the enhancement layer. Consequently, sub8x8partitions are only required to be evaluated in the enhancement layer if these are encoded in the base layer.

\section{3) Only evaluate the base layer list predictions}

A list defines the prediction direction (forward, backward or bi-prediction). It is seen in Fig. 3 that both layers have a high probability for using the same prediction list if the macroblock mode is the same. For MODE_16x16, $\mu_{E L} \in\{1,2,3\}$, it is seen clearly that the same type is used. Consequently, since $\mu_{E L}$ identifies the prediction list, the same prediction list as the base layer has a higher probability. For MODE_16x8 and MODE_8x16 a diagonal of higher probabilities is seen. This diagonal corresponds to $\mu_{B L}=\mu_{E L}$, so the base layer list prediction is favored.

This property can be exploited, due to the resemblance of the video content in both layers. Since the prediction direction is dependent on the video content, both layers are likely to have the same prediction list, because the content in both layers is similar. Therefore, the correspondence between the current frame and the reference frame for both layers will be similar. Consequently, the encoder will most likely use the same prediction direction for both layers.

The presented techniques will result in a lower complexity for the encoder, since the mode decision process does not need to evaluate all macroblock modes, reduce evaluations for submacroblock partitions, and does not have to evaluate all prediction directions. However, each of these properties yield a low loss in bandwidth and quality, since it might be that the

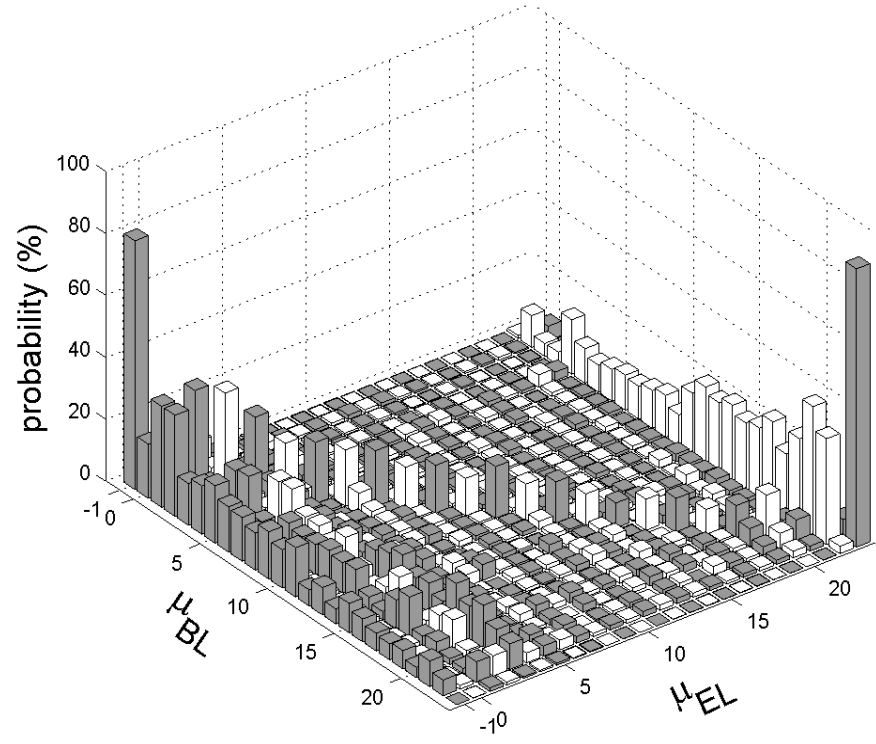

Fig. 3 Average conditional probability identifying the list prediction correlation between both layers.

most optimal enhancement layer mode is not selected. Therefore, combining these three techniques will lower the complexity, but also the RD-preformance. In the next section we will evaluate the consequences of the proposed techniques for complexity and $\mathrm{RD}$, both as standalone techniques and combined with a state-of-the-art fast mode decision model.

\section{RESULTS}

The proposed techniques have been implemented in JSVM 9.4 [12] both as standalone and as additional techniques to Li's model. Four test sequences with different characteristics (Harbour, Ice, Rushhour and Soccer), have been encoded with varying combinations of $Q P_{B L}, Q P_{B L} \in\{18,24,30,36\}$. Dyadic spatial scalability is applied. Two resolution combinations have been tested, QCIF/CIF and CIF/4CIF.

For reference purposes, the test sequences have been encoded with the JSVM reference software. Furthermore, these sequences are encoded with the original encoder optimized with the proposed techniques, with Li's model improved with the proposed techniques and with selective inter-layer residual prediction [5].

The encoded sequences are analyzed for $\mathrm{RD}$ and complexity. The RD measurements are expressed as a difference in bit rate $(\triangle B R)$ and a difference in PSNR $(\triangle \mathrm{PSNR})$ respective to the original encoded sequences. Comparison of the complexity is done by the time saving, given by: $T S=\left(\right.$ Time $_{J S V M}-$ Time $\left._{\text {Fast }}\right) /$ Time $_{J S V M}$. Since the same codebase is used for the original encoder, Li's optimized encoder and all proposed techniques, the difference in time saving gives an indication of the complexity reductions due to our proposed generic techniques.

Time measurements are executed on a dedicated machine with a dual quad core processor and 32 GB of RAM memory. To compare the complexity reduction of the proposed techniques independently of the hardware, the time saving is expressed as a percentage. 
TABLE II

RD AND TIME SAVING RESULTS FOR STANDALONE SCENARIO OF THE PROPOSED TECHNIQUES

\begin{tabular}{|l|c|c|c|}
\hline Method & $\Delta$ BR(\%) & $\Delta$ PSNR(dB) & TS (\%) \\
\hline 1 & 0.60 & -0.05 & 26.95 \\
\hline 2 & $0 . ` 20$ & -0.03 & 53.98 \\
\hline 3 & 0.91 & -0.06 & 17.76 \\
\hline $\mathbf{1}+\mathbf{0 . 5 3}$ & $\mathbf{- 0 . 0 9}$ & $\mathbf{7 3 . 9 1}$ \\
\hline $\mathbf{1 + 2 + 3}$ & $\mathbf{1 . 0 6}$ & $\mathbf{- 0 . 1 3}$ & $\mathbf{7 7 . 1 5}$ \\
\hline
\end{tabular}

$\triangle \mathrm{BR}=$ bit rate increase; $\triangle \mathrm{PSNR}=$ difference in quality (negative means reduction); TS (time saving) =complexity reduction for encoding the enhancement layer.

\section{A. Generic techniques as a standalone solution}

TABLE II shows the average results for the proposed techniques. When only using one improvement (singular techniques), the sub8x8 reduction method (2) results in the highest time saving, virtually without RD degradation. When small complexity reductions are sufficient, this is a good candidate. Only disallowing orthogonal macroblock modes (1) or limiting the list predictions (3) will perform worse for both compression efficiency and complexity.

Obviously, extending technique 2 with 1 results in even better time savings. This performs better than combining 2 with 3, which can be derived from the singular techniques because 3 yields a higher complexity for a more degraded compression performance.

Combining all three techniques will have the highest time saving; however, a bit rate increase of about $1 \%$ has to be acceptable. Improving an original encoder with these three techniques requires only $22 \%$ of the complexity of the original encoder, while nearly an equal compression performance is achieved.

Fig. 4 shows the coding efficiency for the proposed generic techniques used as standalone techniques. It can be seen that only the combination of all techniques has a slightly lower RD performance. Such a small decrease justifies the use of low complex generic techniques. When even lower complexities are required, these techniques can be combined with fast mode decision models. However, the RD will further decrease.

\section{B. Generic techniques to extend fast mode decision models}

While the proposed singular techniques are useful in standalone scenarios, they can also be combined with existing fast mode decision models. We use Li's model to evaluate the effects of the generic improvements for existing fast mode decision models. Since in literature $\mathrm{Li}$ is referred by other models refer, $\mathrm{Li}$ can be seen as a common ground for comparison, e.g in [6] their reported time saving is $7 \%$ lower than $\mathrm{Li}$, while for the combination of their model with $\mathrm{Li}$, the time saving is improved with $11 \%$ compared to $\mathrm{Li}$.

Results for combining Li with our techniques can be found in TABLE III. When using multiple generic techniques, only the results for $\mathrm{Li}+1+2$ are shown, because $\mathrm{Li}+2+3$ and $\mathrm{Li}+1+3$ yield a higher complexity and lower coding efficiencies, for the same reason as with the standalone techniques. Note that while adding one single technique only seems to yield small
TABLE III

RD AND TIME SAVING OF THE PROPOSED TECHNIQUES IN COMBINATION WITH LI'S MODEL [9]

\begin{tabular}{|l|c|c|c|}
\hline Method & $\Delta$ BR(\%) & $\Delta$ PSNR(dB) & TS (\%) \\
\hline $\mathrm{Li}$ & 1.40 & -0.25 & 66.76 \\
\hline $\mathrm{Li}+1$ & 1.55 & -0.27 & 68.37 \\
\hline $\mathrm{Li}+2$ & 1.39 & -0.28 & 82.02 \\
\hline $\mathrm{Li}+3$ & 2.13 & -0.30 & 71.93 \\
\hline $\mathrm{Li}+\mathbf{1}+\mathbf{2}$ & $\mathbf{1 . 5 0}$ & $\mathbf{- 0 . 3 1}$ & $\mathbf{8 4 . 4 7}$ \\
\hline $\mathrm{Li}+\mathbf{1}+\mathbf{2 + 3}$ & $\mathbf{2 . 1 4}$ & $\mathbf{- 0 . 3 6}$ & $\mathbf{8 7 . 2 7}$ \\
\hline
\end{tabular}

time savings, the absolute gains are comparable to those shown in TABLE II. As can be seen, $\mathrm{Li}+1+2$ has only $2.6 \%$ less time gain compared to $\mathrm{Li}+1+2+3$, although the absolute complexity of the latter is $17 \%$ lower compared to the former.

Comparing TABLE II with the results for Li's model, without improvements, show that generic techniques yield better rate distortion (RD) for comparable time savings. From this observation, it can be concluded that singular generic techniques are preferred for small complexity reductions $(<80 \%)$, while the criterion for combinations with fast mode decisions should lie with very low complexity solutions.

In Fig. 6 and Fig. 7, RD-curves for combinations with Li's model are shown for sequence Harbour using a CIF/4CIF resolution, and for sequence Rushhour with a QCIF/CIF resolution. For both resolutions, combining all generic techniques in a standalone scenario outperforms the scenario where only Li's model is applied. Compared to Li's model, improving it with all proposed generic techniques, only slightly degrades the RD performance, while further halving the required complexity. This scenario degrades the picture quality with $0.36 \mathrm{~dB}$ for the PSNR, and requires only an increase of $2.14 \%$ in bandwidth. Compared to Li's model this is only an increase of $0.74 \%$ in bit rate and merely $0.11 \mathrm{~dB}$ lower PSNR. These results satisfy the requirements for using fast mode decision models in real-world systems. However, if only small bit rate increases are allowed, one of the other proposed techniques can be chosen, while the highest possible $\mathrm{RD}$ efficiency is guaranteed.

\section{Improving with selective inter-layer prediction}

As already mentioned in Section I, selective inter-layer residual prediction [5] can be used to extend existing fast mode decision models, since the proposed method evaluates all macroblock modes. In a sense, this makes selective interlayer residual prediction also a generic technique. To stress the universality of generic techniques, Li's model is further improved with selective inter-layer residual prediction. Fig. 5 represents the RD performance for applying selective interlayer residual prediction for Li's model both with and without our proposed generic techniques. As can be seen from this figure and TABLE IV, using selective inter-layer residual prediction with only Li's model results in the same complexity reduction as $\mathrm{Li}+3$, while a slightly better $\mathrm{RD}$ is achieved. Combining selective inter-layer residual prediction with all 


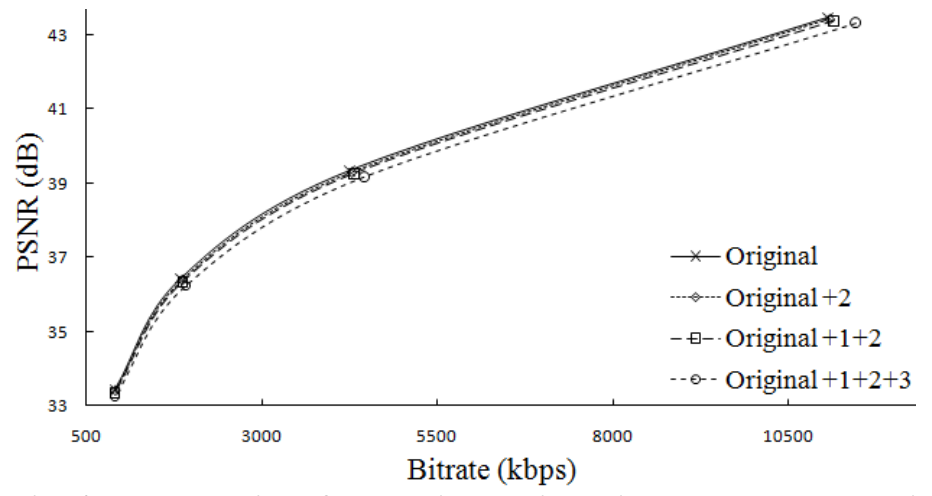

Fig. 4 RD comparison for generic techniques in a standalone scenario for sequence Soccer $@ \mathrm{QP}_{\mathrm{BL}}=\mathbf{3 6}$.

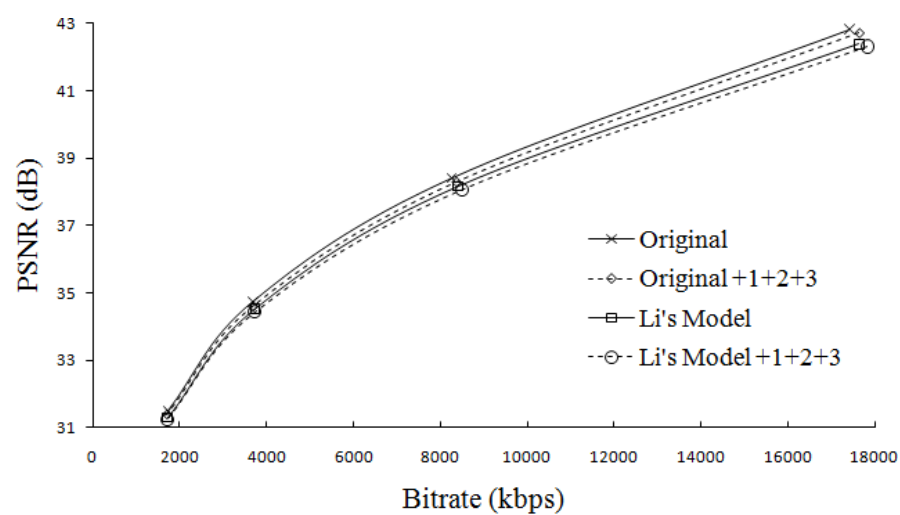

Fig. 6 RD comparison for Harbour @ $Q P_{\mathrm{BL}}=30$ (CIF/4CIF) using the combination of all techniques for both the original and Li's Model.

generic techniques further reduces the complexity (compared to $\mathrm{Li}+1+2+3$, the complexity reduces with $11.5 \%$ ), on the other hand the RD further degrades.

\section{FUTURE WORK}

Depending on the required complexity reduction, one of the above techniques can be used. For systems with a known complexity reduction (e.g.: fixed number of encoded streams), one of the above techniques can be implemented, such that the highest $\mathrm{RD}$ is guaranteed.

When a system with varying complexity is designed, all of the above techniques can be implemented. However, only those necessary to achieve the required complexity should be used to guarantee the highest possible RD performance. This can be done on a per macroblock basis, based on the current actual load. This makes the encoder a complexity scalable encoder. Moreover, complexity scalability schemes can be investigated, not only based on the current load, but also taking into account power consumption, heat dissipation, ... ultimately leading to a green encoder.

\section{CONCLUSION}

The proposed generic techniques are usable in a standalone scenario where a complexity reduction is required, while a high coding efficiency is important. It is shown that these techniques yield a high compression efficiency, independently

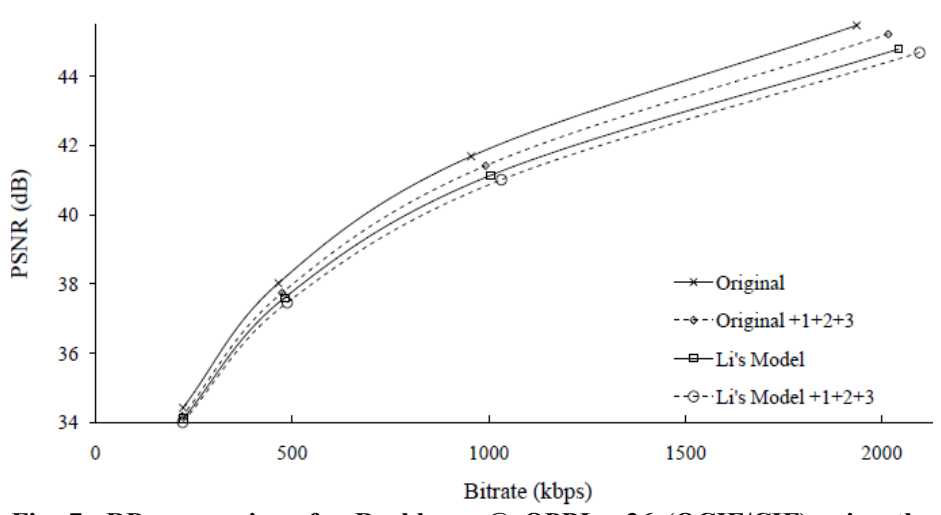

Fig. 7 RD comparison for Rushhour @ QPBL= 36 (QCIF/CIF) using the combination of all techniques for both the original and Li's Model.

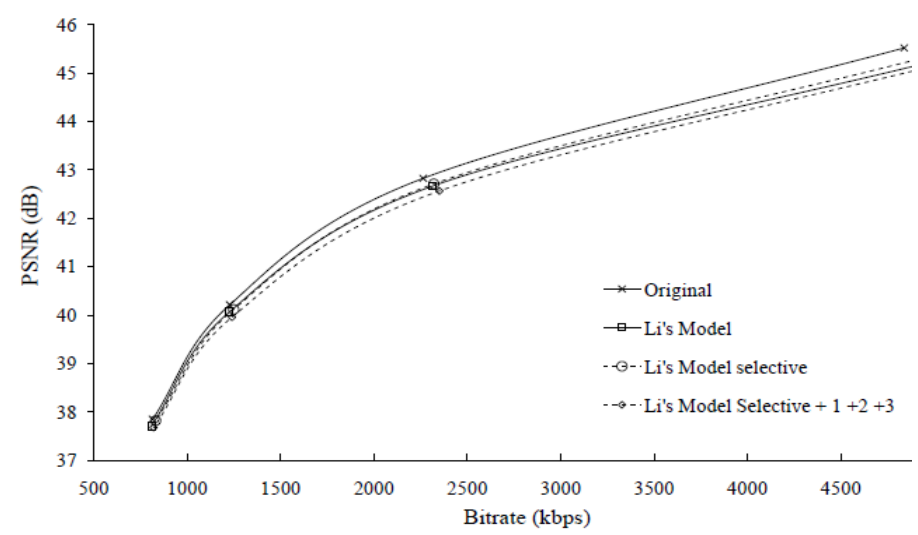

Fig. 5 RD comparison for Ice $@ Q P B L=24(C I F / 4 C I F)$ with selective interlayer residual prediction.

TABLE IV

RD AND TIME SAVING OF SELECTIVE INTER LAYER RESIDUAL PREDICTION IN COMBINATION WITH LI'S MODEL [9]

\begin{tabular}{|l|c|c|c|}
\hline Method & $\Delta \mathbf{B R}(\mathbf{\%})$ & $\Delta$ PSNR(dB) & TS (\%) \\
\hline $\mathrm{Li}+$ Selective & 3.03 & -0.14 & 72.24 \\
\hline $\mathrm{Li}+1+2+3+$ Selective & 3.77 & -0.26 & 88.74 \\
\hline
\end{tabular}

of the content or resolution. When combining these generic techniques with existing fast mode decision models, a system that requires only $12 \%$ of the complexity compared to a normal SVC encoder can be built. Furthermore, we have shown that these techniques can be used with existing optimizations, such as selective inter-layer residual prediction. The latter requires an even lower complexity of $11.5 \%$.

The RD performance degrades for lower complexities, which has to be taken into account when deciding the complexity of the total system. Since the techniques can be applied on a per macroblock basis, the complexity of the encoder can be scaled according to the actual requirements of the system, while always guaranteeing the highest possible RD for any given complexity.

Finally, the presented techniques are compatible with future improved fast mode decision models. This opens the path for the introduction of SVC encoders to allow efficient transport systems to deliver one single bitstream, carrying multimedia content for different types of end-user terminals over heterogeneous networks. 


\section{REFERENCES}

[1] Joint Video Team (JVT) of ISO/IEC MPEG \& ITU-T VCEG, "Advanced video coding for generic audiovisual services, ITU-T Rec. H.264 and ISO/IEC 14496-10 Advanced Video Coding, Edition 5.0 (incl. SVC extension)," Tech. report, MPEG / ITU-T, March 2010.

[2] H. Schwarz, D. Marpe, and T. Wiegand, "Overview of the scalable video coding extension of the H.264/AVC standard," IEEE Trans. on Circuits and Systems for Video Technology, vol. 17, no. 9, pp. 11031120, Sept. 2007.

[3] C. Segall and G. Sullivan, "Spatial scalability within the H.264/AVC scalable video coding extension," IEEE Trans. on Circuits and Systems for Video Technology, vol. 17, no. 9, pp. 1121 -1135, Sept. 2007.

[4] G.Goh, J. Kang, M.Cho, and C.-S. Cho, "Fast mode decision for scalable video coding based on neighboring macroblock analysis", in Proceedings of the 2009 ACM symposium on Applied Computing, pp 1845-1846, March 2009

[5] C.-S. park, S.-J. Baek, M.-S. Yoon, H.-K. Kim, and S.-J. Ko, "Selective inter-layer residual prediction for SVC-based video streaming," IEEE Trans. on Consumer Electronics, vol. 55, no.1, pp. 235-239, Feb. 2009

[6] S.-T. Kim, K. R. Konda, C. Su Park, C.-S. Cho, and S.-J. Ko, "Fast mode decision algorithm for inter-layer coding in scalable video coding," IEEE Trans. on Consumer Electronics, vol. 55, no. 3, pp. 1572-1580, Aug. 2009.

[7] S.-W. Jung, S.-J. Baek, C.-S. Park, and S.-J. Ko, "Fast mode decision using all-zero block detection for fidelity and spatial scalable video coding," IEEE Trans. on Circuits and Systems for Video Technology, vol.20, no. 2, pp. 201-206, Feb.2010.

[8] J.Ren, and N. Kehtarnavaz, "Fast adaptive early termination for mode selection in H.264 scalable video coding," Journal of Real-Time Image Processing, vol.4, no.1, pp. 13-21, Mar.2009

[9] H. Li, Z. Li, C. Wen, and L.-P. Chau, "Fast mode decision for spatial scalable video coding," in International Symposium on Circuits and Systems (ISCAS), May 2006

[10] S. Van Leuven, K. De Wolf, P. Lambert, and R. Van de Walle, Probability analysis for macroblock types in spatial enhancement layers for SVC," in Proceedings of the 11th IASTED International Conference on Signal and Image Processing, pp. 221-227, Aug. 2009

[11] S. Van Leuven, G. Van Wallendael, K. De Wolf, J. De Cock, P. Lambert, and R. Van de Walle, "An enhanced fast mode decision model for spatial enhancement layers in scalable video coding," in Springer Multimedia Tools and Applications, 2010, Accepted for future Publication

[12] Joint Video Team (JVT) of ISO/IEC MPEG \& ITU-T VCEG, "Doc. JVT-W203: Joint Scalable Video Model 10," MPEG / ITU-T, Tech. Rep., April 2007.

\section{BIOGRAPHIES}

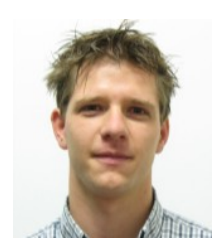

Sebastiaan Van Leuven (S'11) received the M.Sc. degree in Applied Engineering from the University College of Antwerp, Antwerp, Belgium, in 2006 and the M.Sc. degree in Computer Science Engineering from Ghent University, Ghent, Belgium in 2008. Currently, he is with Multimedia Lab, Ghent University, where he is working towards a Ph.D., with the financial support of the Agency for Innovation by Science and Technology (IWT). His main research topic is video coding, including scalable video coding, adaptation of video streams, transcoding and next generation video compression.

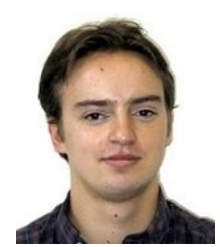

Jan De Cock (M'06) obtained the M.S. and Ph.D. degrees in Engineering from Ghent University, Belgium, in 2004 and 2009, respectively. Since 2004 he has been working at Multimedia Lab, Ghent University, and the Interdisciplinary Institute for Broadband Technology (IBBT). In 2010, he obtained a Post-doctoral Research Fellowship from the Agency for Innovation by Science and Technology (IWT) His research interests include video compression and transcoding, scalable video coding, and multimedia applications.

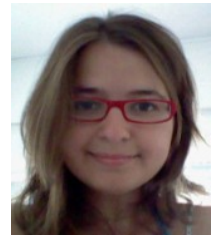

Rosario Garrido-Cantos (S'09) received the M.Sc. degree in Telecommunication Engineering from European University of Madrid, Spain, in 2007 and the M.Sc. degree in Computer Science from University of Castilla-La Mancha, Albacete, Spain, in 2010. From 2004 to 2008 she worked in several companies such as Telefónica I $+\mathrm{D}$, Nokia Siemens Networks, and Accenture. In 2009 she joined the Albacete Research Institute of Informatics, University of Castilla-La Mancha, where she is working towards a Ph.D.. Her research interests include scalable video coding, and video transcoding. She has also been an exchange student at Cork Institute of Technology, Ireland and a visiting researcher at Ghent University, Belgium.

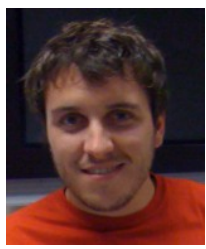

José Luis Martínez (M'07) received his M.Sc. and $\mathrm{Ph} . \mathrm{D}$. degrees in Computer Science and Engineering from the University of Castilla-La Mancha, Albacete, Spain in 2007 and 2009 respectively. In 2005, he joined the Department of Computer Engineering at the University of Castilla-La Mancha, where he was a researcher of Computer Architecture and Technology group at the Albacete research institute of informatics (I3A). In 2010, he joined the department of Computer Architecture of Complutense University in Madrid where he is currently assistant professor. His research interests include Distributed Video Coding (DVC), multimedia standards, video transcoding, and parallel video processing. He has also been a visiting researcher at the Florida Atlantic University, Boca Raton (USA) and Centre for Communication System Research (CCSR), at the University of Surrey, Guildford (UK). He has over 40 publications in these areas in international refereed journals and conference proceedings. He is a member of the IEEE.

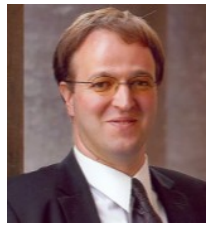

Rik Van de Walle (M'99) received his M.Sc. and PhD degrees in Engineering from Ghent University, Belgium in 1994 and 1998 respectively. After a visiting scholarship at the University of Arizona (Tucson, USA), he returned to Ghent University, where he became professor of multimedia systems and applications, and head of the Multimedia Lab. His current research interests include multimedia content delivery, presentation and archiving, coding and description of multimedia data, content adaptation, and interactive (mobile) multimedia applications. 